\title{
Caídas en adultos mayores institucionalizados: Descripción y evaluación geriátrica
}

\author{
Homero Gac E, Pedro Paulo Marín L, Sergio Castro H, \\ Trinidad Hoyl M, Eduardo Valenzuela A.
}

\author{
Falls in institutionalized \\ elderly subjects. \\ Features and geriatric assessment
}

\begin{abstract}
Background: Falls are an important cause of disability and mortality among elderly subjects. Aim: To study the features and incidence of falls in institutionalized elderly subjects. Patients and methods: Prospective recording of all falls, occurring in a period of eight months, to 453 subjects older than 60 years, living a home for the elderly. Evaluation of functional status of subjects suffering falls, using the Spanish Red Cross score. One hundred and two subjects living in the same place, but not suffering falls, were considered as controls. Results: One hundred and three subjects, aged $80 \pm 6$ years ( $24 \%$ of the sample) suffered falls. Most falls were during the day and while walking. Seventy percent of subjects suffering falls did not have a history of previous falls. Nine percent of those falling, suffered a fracture. Compared to controls, falling subjects had a more deteriorated functional and mental status and consumed more benzodiazepines and neuroleptics. Conclusions: There is an association between functional status and psychotropic medication consumption and the incidence of falls in institutionalized elderly subjects (Rev Méd Chile 2003; 131: 887-94).
\end{abstract}

(Key Words: Accidental falls; Aged; Frail elderly)

Recibido el 21 de agosto, 2002. Aceptado en versión corregida el 19 de junio, 2003.

Programa de Geriatría y Gerontología, Departamento de Medicina Interna, Facultad de Medicina, Pontificia Universidad Católica de Chile. Santiago, Chile

L a población de adultos mayores (AM) en muestro país es de alrededor de 1.300 .000 personas, lo que corresponde a $9,0 \%$ de la población total (según censo de 1992) y se proyecta que para el año 2025 será $3.600 .000^{1}$, por lo que cada

Correspondencia a: Dr. Homero Gac E. Hospital Clínico Pontificia Universidad Católica de Chile. Marcoleta $3475^{\circ}$ piso. Fonos: 3543491-09 8951908. Fax: 6339820. E mail: hgac@med.puc.cl vez es de mayor importancia el adecuado conocimiento de los problemas biomédicos-sociales que afectan a este grupo de personas.

Internacionalmente se ha comunicado que, en los adultos mayores que consultan en un policlínico general, hay cuatro síndromes que con frecuencia los médicos no pesquisan. Estos son los llamados sgigantes de la geriatría: incontinencia uninaria, demencia, inmovilidad y caídas»r. La caída definida como un evento involuntario que precipita a la persona a un nivel inferior 0 al suelo» es un 
problema frecuente en los AM. La razón de una caída rara vez es única y la mayoría de las veces la causa es multifactorial, constituyendo un síndrome clínico. Se estima que la incidencia anual de caídas en el anciano joven (65-70 años) es de $25 \%$ y llega a 35-45\% al tener edad más avanzada (80-85 años), pero superados los 85 años el número de caídas reportadas disminuye, posiblemente por restricción de la actividad física. Se ha comprobado que los AM frágiles se caen más que los vigorosos ( $52 \%$ vs $17 \%$ ), aunque las consecuencias de las caídas son más graves en estos últimos ${ }^{3-10}$. Las únicas publicaciones nacionales sobre caídas en AM, son la revisión de L Cartier ${ }^{11}$ y el estudio que se realizó en AM que están en control ambulatorio de $\mathrm{G}$ González et al, quien comunica 18,2\% de incidencia de caídas en los 6 meses previos a la consulta ${ }^{12}$.

La incidencia reportada de caídas en AM institucionalizados se eleva hasta $50 \%$, con consecuencias graves en $17 \%$ de ellos ${ }^{13}$.

Las instituciones para AM están aumentando en nuestro país y realizan una importante labor en la atención de personas que tienen una amplia gama de limitaciones tanto funcionales como cognitivas. El presente estudio describe las características de las caídas de una población de AM institucionalizados, en cuanto a su frecuencia, circunstancias y consecuencias y además compara la evaluación geriátrica de los sujetos que sufren caídas con aquellos sujetos que no se caen.

\section{Material y MÉTodo}

Se realizó un estudio prospectivo y descriptivo en una población de 453 personas que vivían en ocho residencias de larga estancia para adultos mayores, pertenecientes a la Fundación Las Rosas de Ayuda Fraterna, en Santiago de Chile. De estas residencias, seis eran principalmente para pacientes autovalentes (total 383 personas) y dos correspondían a hogares para personas con deterioro cognitivo (70 residentes).

En el estudio se incluyeron a todos los AM de estas residencias presentes entre marzo y octubre de 1995, registrándose todas las caídas que se produjeron durante ese período. Los datos fuemon registrados en las primeras $24 \mathrm{~h}$ de ocurido el evento por un médico o una enfermera del centro, completándose una ficha común que contenía los datos generales del paciente, hora y lugar de la caída, antecedente de caídas previas y consecuencias de éstas. En el caso de ocumir una lesión grave, el paciente era trasladado al Servicio de Urgencia respectivo y se recolectaron los diagnósticos finales de la epicrisis hospitalaria. Para la evaluación genátrica, se utilizó la Escala de la Cruz Roja Española (funcional y mental) donde se clasifica al sujeto de 0 a 5 puntos (siendo 0 el grado mejoróptimo y 5 puntos equivale a malo o pésimo, Anexo 1) ${ }^{10,11}$. Al final del estudio, aleatoriamente, se eligió un grupo de $102 \mathrm{AM}$ de la misma población que no tuvo caídas (grupo control), para compararlos con aquellos que sí las presentaron.

Los datos fueron analizados usando el programa de computación Epi Info. La comparación de promedios se hizo con la prueba de t de Student y la comparación de porcentajes con la prueba Chi cuadrado.

\section{Resultados}

Las características generales de la población estudiada $(n=453)$ se presentan en la Tabla 1. Durante

Tabla 1. Características generales de la población institucionalizada ( $n=453$ sujetos)

\begin{tabular}{|c|c|}
\hline Sexo & Femenino $383(84,5 \%)$ \\
\hline Edad promedio & $81,2 \pm 7,9$ años \\
\hline Hogar de procedencia & Autovalentes $385(85 \%)$ \\
\hline & Dementes 68 (15\%) \\
\hline \multicolumn{2}{|l|}{ Antecedente de enfermedades: } \\
\hline Hipertensión arterial & $41 \%$ \\
\hline Insuficiencia cardíaca & $21 \%$ \\
\hline Cardiopatía comonaria & $12,3 \%$ \\
\hline Accidente vascular encefálico & $5,8 \%$ \\
\hline Depresión & $13 \%$ \\
\hline Patología osteomuscular & $40 \%$ \\
\hline Diabetes & $7 \%$ \\
\hline Número de fármacos promedio & 2,1 por persona \\
\hline Personas que usan 50 más fármacos & $6,5 \%$ \\
\hline Benzodiacepinas & $38 \%$ \\
\hline Vasodilatadores & $22 \%$ \\
\hline Neurolépticos & $18 \%$ \\
\hline Antidepresivos & $15 \%$ \\
\hline
\end{tabular}


Tabla 2. D escripción de los 113 sujetos que presentaron caídas, según el lugar de residencia

\begin{tabular}{|lcc|}
\hline & $\begin{array}{c}\text { Hogares } \\
\text { No Dementes } \\
(\mathrm{n}=79)\end{array}$ & $\begin{array}{c}\text { Hogares } \\
\text { Dementes } \\
(\mathrm{n}=34)\end{array}$ \\
\hline Mujeres & $66(83,5 \%)$ & $34(100 \%)^{* *}$ \\
Edad (años) DS & $81,8 \pm 7,4$ & $79,7 \pm 9,2$ \\
Rango de edad (años) & 63 a 97 & 57 a 95 \\
Relación y porcentaje & $79 / 38520,5 \%$ & $34 / 7247,2 \%^{*}$ \\
Sujetos con caídas previas & $29(36,7 \%)$ & $4(11,8 \%)^{*}$ \\
Número de caídas previas (X+DS) & $3,2 \pm 2,6$ & $8,5 \pm 7,5^{*}$ \\
Rango de caídas previas & 1 a 12 & 2 a 15 \\
Casos con fracturas & 8 & $2 *$ \\
\hline
\end{tabular}

$*_{p}<0,05 .{ }^{*} p<0,01$.

los ocho meses que duró el estudio, 113 adultos mayores presentaron caídas, lo que corresponde a $24 \%$ de los residentes (113 de 453 sujetos), la edad promedio de este grupo fue $80,3 \pm 6,2$ años. Porcentualmente las mujeres presentaron caídas en $26,1 \%$ (100 de 383 mujeres) y los hombres en 18,5\% (13 de 70). Estos 113 adultos mayores presentaron 207 caídas durante el estudio. La frecuencia de caídas fue: $54,9 \%$ una sola caída, $37,4 \%$ se cayeron dos a cinco veces, $7,3 \%$ lo hizo seis a diez veces y sólo uno $(0,5 \%)$ se cayó quince veces. En el horario diurno (6:00 a 20:00 horas) ocurrieron la mayoría de las caídas ( $\mathrm{n}=166 ; 80,2 \%)$ y también de fracturas (6 de 10 casos).

En la Tabla 2, se describe la frecuencia de caídas según la residencia de origen: 69,9\% vivía en los hogares para autovalentes (79 personas) cuya edad promedio fue $81,8 \pm 7,4$ DS años, y $30,1 \%$ habitaba en hogares para dementes (34 personas), con una edad promedio de $79,7 \pm 9,2$ DS años ( $p$ NS). De los 113 sujetos que se cayeron, $70 \%$ no presentaba caídas previas y $29 \%$ ( $\mathrm{n}=33$ sujetos) sí (promedio 3,8 caídas previas; rango 1 a 15 caídas). De los 33 sujetos con caídas previas (Tabla 2), 29 vivían en hogares para autovalentes, los cuales tenían significativamente ( $p<0,006$ ) menos caídas previas (promedio $3,2 \pm 2,65$ DS) que los cuatro sujetos que vivían en hogares para dementes (promedio 8,5 $\pm 7,5$ DS caídas previas). En los casos con fracturas la diferencia no fue significativa $(p<0,72)$.
En la Tabla 3 se describe el lugar donde ocurrieron las 207 caídas y los 10 casos de fracturas y se aprecia que la mayoría ocurre en los lugares habituales de desplazamiento. Respecto a la situación en que ocurrió la caída, en $64,8 \%$ fue caminando, $24,4 \%$ al levantarse de la cama y 11,8\% al levantarse del sillón.

En la Tabla 4 se presenta la evaluación geriátrica, que incluye el estado funcional $\mathrm{y}$ mental de los 113 sujetos que se cayeron. Se observa que $76,1 \%$ ocurrió en personas sin grandes limitaciones funcionales en el diario vivir (puntaje 0 a 2) y este grupo presentó 9 de las 10 fracturas, siendo significativamente mayor que el grupo con peor estado funcional. Respecto a las condiciones mentales se observa que $56,6 \%$ de las caídas ocurrió en los con menos alteraciones cognitivas (puntaje 0 a 2) donde están 5 de los 10

Tabla 3. Lugar donde ocurrió la caída y la fractura

\begin{tabular}{|lccc|}
\hline Lugar & $\begin{array}{c}\text { Caídas } \\
\mathrm{n}=207\end{array}$ & $\begin{array}{c}\text { Fractura } \\
\mathrm{n}=10\end{array}$ & \% de las caídas \\
\hline Dormitorio-cama & 85 & 4 & 4,7 \\
Baño & 25 & 2 & 8,0 \\
Comedor & 24 & 0 & - \\
Pasillo & 36 & 3 & 8,3 \\
Patio & 23 & 0 & - \\
Otro lugar & 14 & 1 & 7,1 \\
\hline
\end{tabular}


Tabla 4. Evaluación geriátrica de los 113 sujetos que presentaron caídas

\begin{tabular}{|lccccc|}
\hline \multirow{2}{*}{ Puntaje } & \multicolumn{2}{c|}{$\begin{array}{c}\text { Evaluación Funcional } \\
\mathrm{n}\end{array}$} & $\%$ & \multicolumn{2}{c|}{$\begin{array}{c}\text { Evaluación Mental } \\
\mathrm{n}\end{array}$} \\
\hline 0=óptima & 25 & 22,1 & 15 & $\%$ \\
1=buena & 27 & 23,9 & 22 & 19,3 \\
2=más que regular & 34 & 30,1 & 27 & 23,9 \\
3=regular & 11 & 9,7 & 23 & 20,4 \\
4=malo & 12 & 10,6 & 18 & 15,9 \\
5=pésimo & 4 & 3,5 & 8 & 7,1 \\
Grupo 0 a 2 & 86 & 76,1 & 49 & 56,6 \\
Grupo 3 a 5 & 27 & 23,9 & 43,4 \\
\hline
\end{tabular}

Tabla 5. Comparación de la Evaluación Funcional

\begin{tabular}{|lccccc|}
\hline \multirow{2}{*}{ Puntaje } & \multicolumn{2}{c}{ Caen } & n & No caen \\
\hline 0=óptima & 25 & 22,1 & 63 & $\% 1,7$ \\
1=buena & 27 & 23,9 & 16 & 15,6 \\
2=más que regular & 34 & 30,1 & 15 & 14,7 \\
3=regular & 11 & 9,7 & 3 & 2,9 \\
4=malo & 12 & 10,6 & 4 & 3,9 \\
5=pésimo & 4 & 3,5 & 1 & 0,9 \\
Grupo 0 a 2 & 86 & 76,1 & 94 & $92,1^{*}$ \\
Grupo 3 a 5 & 27 & 23,9 & 8 & $7,9^{*}$ \\
\hline
\end{tabular}

${ }_{p}^{*}<0,01 .{ }^{* *} p<0,001$.

casos de fracturas, no encontrando diferencias con el grupo en peor estado mental.

La Tabla 5 muestra la comparación del estado funcional de los pacientes que presentaron caídas $\mathrm{v} / \mathrm{s}$ el grupo control, apreciándose diferencias altamente significativas entre ambos grupos. El grupo control (sin caídas) presenta una mejor situación funcional que aquellos que tuvieron caídas durante el estudio, lo mismo se observa en relación al estado mental (Tabla 6).

Las consecuencias de las 207 caídas fueron: 40\% ninguna, 7,7\% heridas cortantes simples, 35\% contusión simple, $11,2 \%$ hematomas, $1 \%$ traumatismo encefalocraneano, $1 \%$ esguince y 10 casos $(4,9 \%)$ de fracturas, lo que corresponde a $8,8 \%$ de los pacientes que se cayeron. Del total de caídas, 141 ocurrieron en los hogares para autovalentes (con ocho fracturas, 5,7\%) y 65 en los hogares para dementes (dos casos de fracturas, $3,1 \%$ ) sin tener significación $(p=0,42)$. Un paciente falleció a consecuencia de un traumatismo encefalocraneano.

Respecto a las diez personas que presentaron fracturas, la edad promedio fue 82,2 años (rango 75 a 84 años) y cinco de ellos habían presentado caídas previas (rango 1 a 2 veces). Los tipos de fracturas fueron: seis casos de fractura de cuello de fémur, dos de fractura de Colles, una de fractura de piema y una fractura nasal. Del total de casos fracturados, nueve fueron tratados en el hospital. Los lugares donde se fracturaron se presentan en la Tabla 3 y fueron: en el dormitorio cuatro casos $(4,7 \%$ de las caídas en el dormitorio), en el baño dos (8\%) y en el pasillo tres (8,3\%); ocurrieron en el horario diumo (6:00 a 20:00 h) en ocho casos. 
Tabla 6. Comparación de la Evaluación M ental

\begin{tabular}{|lccccc|}
\hline Puntaje & \multicolumn{2}{c}{ Caen } & \multicolumn{2}{c|}{ No Caen } \\
\hline 0=óptima & $\mathrm{n}$ & $\%$ & $\mathrm{n}$ & $\%$ \\
1=buena & 15 & 13,3 & 33 & 32,3 \\
2=más que regular & 22 & 19,5 & 17 & 16,6 \\
3=regular & 27 & 23,9 & 43 & 42,1 \\
4=malo & 23 & 20,4 & 3 & 2,9 \\
5=pésimo & 18 & 15,9 & 5 & 4,9 \\
Grupo 0 a 2 & 8 & 7,1 & 1 & 0,9 \\
Grupo 3 a 5 & 64 & 56,6 & 93 & $91,2^{*}$ \\
& 49 & 43,4 & 9 & $8,8^{* *}$ \\
\hline
\end{tabular}

$*_{p}<0,001 . *{ }^{*} p<0,001$.

En la Tabla 7 se presentan los datos obtenidos sobre el uso de fármacos, encontrándose que el grupo con caídas usaba significativamente más benzodiacepinas y neurolépticos que el grupo control.

De la muestra aleatoria de 102 pacientes que no sufrieron caídas en este período se encontró que $85(83,3 \%)$ eran mujeres y $17(16,7 \%)$ hombres con una edad de promedio 79 años. El 84,2\% procedían de hogares para autovalentes y 15,8\% de hogares para dementes. No hubo diferencias entre este grupo y el total de pacientes en cuanto a prevalencia de enfermedades médicas crónicas.

\section{DISCUSIÓN}

Los accidentes ocupan el séptimo lugar como causa de muerte en los adultos mayores. Las caídas a su

Tabla 7. U so de fármacos de ambos grupos

\begin{tabular}{|lcc|}
\hline & $\begin{array}{c}\text { Pacientes Caídos } \\
\mathrm{n}=113 \\
\text { \% de usuarios }\end{array}$ & $\begin{array}{c}\text { Sujetos Control } \\
\mathrm{n}=102 \\
\text { \% de usuarios }\end{array}$ \\
\hline Benzodiacepinas & 40,7 & $29,4^{*}$ \\
Neurolépticos & 30,9 & $14,7^{*}$ \\
Vasodilatadores & 29,2 & 25,4 \\
Antidepresivos & 20,3 & 20,5 \\
\hline * $<0,05$.
\end{tabular}

vez, son la principal causa de accidentes en este grupo de edad. Aunque frecuentemente las caídas no tienen consecuencias mortales, sí afectan la salud y la calidad de vida de las personas ${ }^{17}$.

Las caídas constituyen uno de los síndromes geriátricos más importantes por su elevada incidencia y especialmente por las repercusiones que va a provocar en la calidad de vida tanto del anciano como del cuidador. Se reporta que un tercio de las personas mayores de 65 años que viven en la comunidad, sufren una caída anual, elevándose esta frecuencia hasta $40 \%$ de los ancianos mayores de 75 años $^{3}$. En los ancianos institucionalizados, dadas las características especiales de este grupo (mayores limitaciones funcionales y pluripatologías), la incidencia aumenta reportándose hasta $50 \%$ anual en los Estados Unidos ${ }^{13-15}$. En ese país hay más de un millón quinientos mil residentes, $\mathrm{y}$ entre 10 a $20 \%$ de las caídas en instituciones producen lesiones serias (2-6\% fracturas). También la población de estas instituciones se ha envejecido, así es como los mayores de 85 años son en promedio $56 \%$ del total de residentes ${ }^{2}$.

Se ha comprobado que los cambios normales del proceso del envejecimiento y las patologías adyacentes, contribuyen al aumento del número de caídas en el AM. Participan en ello factores sensoriales (como la disminución de la percepción de profundidad, la susceptibilidad al deslumbramiento, la disminución de la agudeza visual, y las dificultades en la acomodación), cambios neurológicos (pérdida del equilibrio y de la propiocepción, aumento en el tiempo de reacción), modificaciones 
cardiovasculares (hipotensión postural y otros), cambios mentales (cuadros de confusión, conducta impulsiva) y modificaciones músculo-esqueléticas (posturas incorrectas y disminución de la fuerza muscular) entre los más destacables ${ }^{19}$.

Uno de los factores que determinan la escasa preocupación por las caídas en el público general y en los miembros del equipo de salud, es su denominación como «caídas o accidentes», lo que habitualmente se entiende como un suceso casual 0 fortuito. No obstante, cuando se educa al personal de salud y éste tiene presente las causas, factores predisponentes y consecuencias de las caídas, está demostrado que pueden hacerse intervenciones que logran, al menos parcialmente, disminuir su frecuencia y/o la gravedad de sus consecuencias como son las fracturas, incapacidad funcional y otras ${ }^{20}$. Las caídas, son por su incidencia y gravedad un problema de salud pública importante porque afecta la calidad de vida de la persona y porque provoca un aumento en la dependencia y necesidad de ayuda incluso para las actividades básicas de la vida diaria. Sabemos que en un número elevado de casos las caídas son evitables; controlando la medicación y advirtiendo al anciano de sus riesgos, instruyendo sobre las adaptaciones necesarias especialmente en las instituciones donde reside ${ }^{13,14}$.

En todos los países, un porcentaje de los AM reside en Residencias, llamados también «Hogar de Anciano, Institución Geriátrica, Residencia Geriátrica o Establecimiento Geriátrico», que es un establecimiento destinado fundamentalmente al alojamiento, para mantener el bienestar psíquico y social de la población más envejecida. Es esta población la que tiene un mayor riesgo de presentar caídas, a su vez, cerca de $40 \%$ de las admisiones a una institución es producida por consecuencias secundarias de una caída $^{4,10}$. La población de estas instituciones es, por consiguiente, un grupo seleccionado de alto riesgo de caídas.

La incidencia de caídas en nuestro estudio fue de $24 \%$, cifra que está dentro del límite inferior de lo reportado internacionalmente (varían entre 20\% y 50\%). Esta variabilidad, puede estar dada por la gran diferencia entre las poblaciones analizadas en estos estudios. Cabe destacar, que nuestra población tenía un promedio de edad de 81,2 $2 \pm 7,9$ años y no se puede explicar, por lo tanto, por una menor edad esta diferencia (en hogares de otros países latinoamericanos el promedio de edad es alrededor de 70 años).

En nuestro trabajo no fue posible interpretar las diferencias en la frecuencia de caídas por sexo, dado existe el sesgo de que todos los pacientes de hogares para dementes analizados eran mujeres. En trabajos internacionales se observa una mayor tendencia a caerse en las mujeres, lo cual ha sido atribuido a un mejor estado funcional y mayor movilidad de ellas y a mayor presencia de artrosis y osteoporosis.

Al analizar nuestros datos por procedencia, observamos que $47,2 \%$ de los pacientes dementes presentaron caídas versus 20,5\% del grupo no demente con diferencias significativas entre ambos, lo cual confirma una vez más que las alteraciones cognitivas son un factor de riesgo importante para caídas $^{16}$. Es destacable, sin embargo, que los pacientes no dementes tienen un mayor número de fracturas y mayor número de caídas previas, lo que debiese explicarse por una mayor autonomía y probabilidad de enfrentar riesgos extrínsecos de caídas. Lo anterior se correlaciona con que la mayor parte de las caídas estudiadas fueron cuando los pacientes estaban caminando $(64,8 \%)$.

Respecto al lugar donde acontecen las caídas, el dormitorio y los pasillos parecen ser importantes tanto por el tiempo de permanencia en el primer caso como por el espacio de deambulación continua en el segundo. Especial interés cobran las caídas en el baño, las que son terceras en frecuencia y determinan fracturas en $8 \%$ de los casos. El hecho que la mayor parte de los eventos ocurra en horario diurno (80,2\%), facilita la posibilidad cierta de prevenirlas, ya que el personal del hogar es mayor en número y puede estar más atento en este horario.

Al analizar el estado funcional y mental, se observa que habría un mayor riesgo a medida que el deterioro aumenta, pero se llegaría a un punto en el cual los pacientes dejan de caerse por tener menor movilidad. Esto explicaría el hecho observado que los pacientes que se caen en general tienen un peor estado que aquellos que no lo hacen; pero asimismo los pacientes con los peores estados funcionales y mentales llegan a un punto en que por su deterioro se caen menos.

Al comparar diferencias en el uso de fármacos entre el grupo que presentó caídas y el control, encontramos diferencias significativas en el uso de 
benzodiacepinas y neurolépticos, siendo mayor en el grupo que tuvo caídas; no existió diferencias en uso de antidepresivos y vasodilatadores. Esto puede ser una información útil a la hora de focalizar a aquellos pacientes de mayor riesgo por uso de estos fármacos.

Cabe señalar que debido al nivel socioeconómico bajo de los pacientes no se dispuso de un estudio mayor del origen de las demencias en aquellos que portaban esta patología, habría sido interesante comparar a aquellos con demencias corticales con los afectados por demencias subcorticales, los cuales uno supone más propensos a caídas. Asimismo futuros estudios pudieran contemplar análisis de otras alteraciones neurológicas evaluadas por especialista como polineuropatías, alteraciones frontales o trastomos cerebelosos, etc; los que pudieran incidir en la presencia de caídas.

Finalmente, en relación a las consecuencias de las caídas encontramos que $8,8 \%$ de los pacientes presentaron fracturas y que un paciente falleció por un traumatismo encefalocraneano. Se han descrito ciertos factores de riesgo, que pueden modificarse para lograr prevenir las fracturas que son: suplementos de calcio y vitamina D (97\% tienen ingesta debajo de la cantidad diaria recomendada y una de tres mujeres institucionalizadas es muy deficiente en vitamina D), plan de ejercicio diario y protectores externos de cadera ${ }^{20}$. Las caídas son causadas por la interacción de un número de diversos factores. Por esta razón, la prevención de la caída requiere una combinación del tratamiento médico, de la rehabili-

\section{REFERENCIAS}

1. Mardones F, Javet L, Valdivia G. El desafío del cambio demográfico en Chile. Boletín de la Escuela de Medicina P Universidad Católica de Chile, 2000; 29: 5-9.

2. Kane RL, Oustander JG, Abass IB. Essentials of Clinical geriatrics 3 ${ }^{\text {rd }}$ Ed. New York: Mc Graw-Hill, 1994.

3. GAC H. Caídas e Inmovilidad. Boletín de la Escuela de Medicina P Universidad Católica de Chile, 2000; 29: 18-22.

4. Rubenstein LZ, Josephson KR, Robiins AS. Falls in the nursing home. Ann Intern Med 1994; 121: 442-51.

5. Rynanen OP, Kiveia SL, Honkanen R. Times places and mechanism of falls among the elderly. Gerontology 1991; 24: 154-61. tación, de la modificación ambiental, y de uso de cierta tecnología.

Las intervenciones más conocidas incluyen:

- Ejercicios regulares y/o rehabilitación orientada a mejorar la fuerza y resistencia, terapia física, entrenamiento del paso, o programas del caminar.

- Gravámenes ambientales y modificaciones para mejorar movilidad y seguridad (ej: instalando barras en las murallas, agregando asientos levantados del WC, bajando las alturas de la cama, instalando barandillas en los vestíbulos). Revisión constante de los fármacos prescritos, para determinar sus riesgos y potenciales ventajas.

- Dispositivos tecnológicos (eg, sistemas de alarma que se activan cuando los pacientes intentan salir de la cama o moverse sin ayuda, 0 cojines protectores de la cadera) $)^{3,14,20}$.

Creemos que los datos obtenidos de este trabajo muestran la alta significación del problema y permiten focalizar los recursos para que pudieran reducirse los eventos en horario diurno, mejorando los cuidados en estas horas y optimizando aquellos factores extrínsecos en dormitorios y pasillos que pudiesen ocasionar más caídas. Asimismo evitar caídas, especialmente en aquellos pacientes con deterioro funcional y mental moderado y en los usuarios de psicofármacos como neurolépticos y benzodiacepinas.

6. Visentin P, Ciravegna R, Uscello L, Molaschi M, Fabris F. Site-specific relative risk of fractures in the institutionalized eldenly. Gerontology 1995; 41: 273-9.

7. CaL CM, KIel DP. An epidemiological study of fall-related fractures among institutionalized older people. J Am Geriatr Soc 1995; 43: 1336-40.

8. Marín PP, Valenzuela E, Reyes P. Evaluación geriátrica de adultos mayores en un Hospital Universitario. Rev Méd Chile 1994; 122: 1362-6.

9. Franzoni S, Rozzoni R, BofFe山 S, Frisoni GB, TRABUCCH $M$. Fear of falling in nursing home patient. Gerontology 1994; 40: 38-44.

10. VIsKum B, JuUL S. Accidental falls in nursing homes. A study on the role of drugs in accidental falls in nursing homes. Ugeskr-Laeger 1992; 154: 2955-8. 
11. CaRTier LR. Caídas y alteraciones de la marcha en los adultos mayores. Rev Méd Chile 2002; 130: 332-7.

12. González G, Marín PP, Pereira G. Características de las caídas en el adulto mayor que vive en la comunidad. Rev Méd Chile 2001; 129: 1021-30.

13. Calvo JJ. Caídas en la comunidad y en instituciones. Grupo de trabajo de caídas de la Sociedad Española de Geriatría y Gerontología. En: Evaluación del anciano con caídas a repetición. Madrid; Editorial Fundación Mapfre Medicina. 1997; 3-11.

14. TinetTi ME, Lu WL, GinTER SF. Mechanical restraint use and fall-related injuries among residents of skilled nursing facilities. Ann Intern Med 1992; 116: 369-74.

15. Kanten DN, Mulrow CD, Gerety MB, Lichtenstein MJ, Aguilar C, CoRNeLl JE. Falls: an examination of three reporting methods in nursing homes. J Am Geriatr Soc 1993; 41: 662-6.
16. Van Dijk PT, Meulenberg OG, Van De Sande HJ, HABBEMA JD. Falls in dementia patients. Gerontologist 1993; 33: 200-4.

17. OLVVer M. Consecuencias médicas: mortalidad y morbilidad de las caídas. Grupo de trabajo de caídas de la Sociedad Española de Geriatría y Gerontología, En: Evaluación del anciano con caídas a repetición. Madrid; Editorial Fundación Mapfre Medicina. 1997; 3-11.

18. EJAZ FK, JonES JA, Rose MS. Falls among nursing homes residents: an examination of incident reports before and after restraint reduction programes. J Am Geriatr Soc 1994; 42: 960-4.

19. Marín PP, GAC H. Cambios asociados al envejecimiento. Boletín de la Escuela de Medicina P Universidad Católica de Chile, 2000; 29: 11-4.

20. Grisso JA, Kelsey JL, Strom BL, Chin GY, Maislin G, O’BRIEN LA ET AL. Risk factors for falls as a cause of hip fractures in women. N Engl J Med 1991; 324: 1326-31.

\section{ANEXo 1 \\ Escala de la CRUZ Roja de InCAPACIDAD}

En España también se usa un índice global de incapacidad física desarrollado por la Cruz Roja.

Grados de incapacidad física

0 . Se vale totalmente por sí mismo, anda con normalidad.

1. Realiza suficientemente los actos de la vida diaria. Deambula con alguna dificultad, continencia total.

2. Tiene alguna dificultad en los actos diarios, por lo que en ocasiones necesita ayuda. Deambula con ayuda de bastón o similar. Continencia total o rara incontinencia.

3. Grave dificultad en bastantes actos de la vida diaria. Deambula difícilmente ayudado al menos por una persona. Incontinencia ocasional.

4. Necesita ayuda para casi todos los actos. Deambula ayudado con extrema dificultad (2 personas). Incontinencia habitual.

5. Inmovilizado en cama o sillón. Incontinencia total. Necesita cuidados continuos de enfermería.

Grados de incapacidad psíquica

0 . Totalmente normal.

1. Algunas «rarezas», ligeros trastornos de desorientación en el tiempo. Se puede hablar con él «cuerdamente».

2. Desorientación en el tiempo. La conversación es posible, pero no perfecta. Conoce bien a las personas, aunque a veces olvide alguna cosa. Trastornos de carácter, sobre todo si se le disgusta. Incontinencia ocasional.

3. Desorientación. Imposible mantener una conversación lógica. Confunde las personas. Claros trastornos del humor. Hace cosas que no parecen explicables a veces o a temporadas. Frecuente incontinencia.

4. Desorientación. Claras alteraciones mentales que la familia o el médico han etiquetado ya de demencia. Incontinencia habitual total.

5. Demencia senil total, con desorientación de las personas, etc. Vida vegetativa agresiva o no. Incontinencia total. 